\title{
Optical processor arrays for controlling focal length or for tuning the depth of field
}

\author{
J. Ojeda-Castañeda* and C. M. Gómez-Sarabia \\ Electronics Department, University of Guanajuato, Salamanca, 36885, Guanajuato, México \\ Received January 26, 2011; accepted March 30 2011; published March 31, 2011
}

\begin{abstract}
We present two families of optical processor arrays. One family uses a pair of complex conjugate phase arrays for implementing a varifocal lenslet array, which is useful for axial scanning. The other family uses a different pair of complex conjugate phase arrays for controlling the depth of field in a telecentric processor array.
\end{abstract}

By using a suitable pair of nonconventional optical elements, it is possible to implement a lens, whose focal length can be modified [1-3]. A similar type of optical elements can be applied for generating wavefront aberrations $[4,5]$. And a rather similar approach can be applied for implementing in a tunable fashion the phase masks for extending the depth of field [6, 7]. It is worthwhile to note that the same ideas have a counterpart when using Moiré diffractive elements. By using the latter approach, Burch and Williams rediscovered Alvarez's proposal [8], by improving on the Lohmann and Paris method [9]. Further advances in this field are reported in references [10-12].

Our aim here is to unveil two families of telecentric processor arrays. One family set employs a pair of suitable phase masks for implementing a lenslet array with a variable focal length. We show that this feature is useful for multiple axial scanning. And the other family set uses a different pair of masks for tuning the depth of the field of a telecentric processor array.

We discuss our proposal in simple heuristic terms. Then, we unveil an optical processor array that uses a pair of suitable phase masks (at the Fourier plane) for multiple axial scanning. Eventually, we show that another pair of masks is useful for tuning the depth of the field in a lenslet array.

Figure 1 shows schematically a pair of 2-D arrays, each array has the same number of elements, say $\mathrm{N} \times \mathrm{N}$. As depicted in Fig. 1b), at each element there is a phase mask, whose complex amplitude transmittance is

$t_{1}(x, y)=\exp (i \varphi(x, y)) \operatorname{rect}(x / \varepsilon d) \operatorname{rect}(y / \varepsilon d)$.

*E-mail: jorge_ojedacastaneda@yahoo.com
In Equation 1, we denote as $\varphi(\mathrm{x}, \mathrm{y})$ the phase delay; the Latin letter $d$ denotes the distance between any two consecutive elements along either the $\mathrm{x}$-axis or the $\mathrm{y}$-axis; and the Greek letter $\varepsilon$ is the fill factor, $0<\varepsilon<1$, at any cell. In Fig. 1c) we show a second array. Now, as is depicted in Fig. 1d), at each element of the array, the complex amplitude transmittance is

$t_{2}(x, y)=\exp (-i \varphi(x, y)) \operatorname{rect}(x / \varepsilon d) \operatorname{rect}(y / \varepsilon d)$.

In other words, the complex amplitude transmittance in Eq. (2) is the complex conjugate of the amplitude transmittance in Eq. (1).
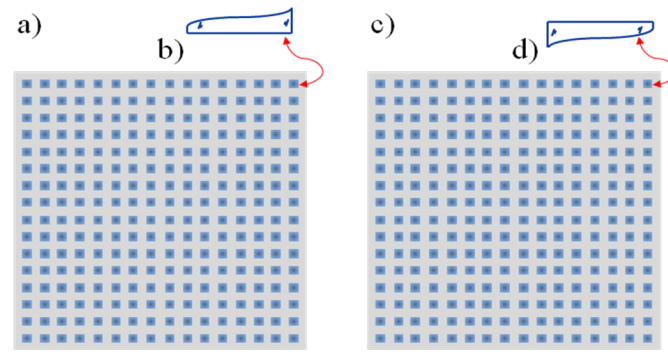

Fig. 1. Schematics of a 2-D array, and its phase conjugated screen.

Here we propose to use the above two phase screens as a pair, while introducing a lateral displacement, $s$, between them. Hence, the complex amplitude transmittance of the pair is

$$
\begin{aligned}
t(x, y ; s)= & t_{1}(x+s / 2, y) t_{2}(x-s / 2, y) \\
= & \exp \{i[\varphi(x+s / 2, y)-\varphi(x-s / 2, y)]\} \\
& \operatorname{rect}[x /(\varepsilon-s / d) d] \operatorname{rect}(y / \varepsilon d) .
\end{aligned}
$$

Figure 2 illustrates the operation in Eq. (3), by using a lateral view of the above arrays. Along line $2 a$ ) we show an asymmetrical phase profile; while along line $2 b$ ) we display its complex conjugate screen. The use of these two complex conjugate masks as a pair, with $\mathrm{s}=0$, is illustrated in Fig. 2c). And in Fig. 2d), we show the use of the same pair, but with $\mathrm{s} \neq 0$. 


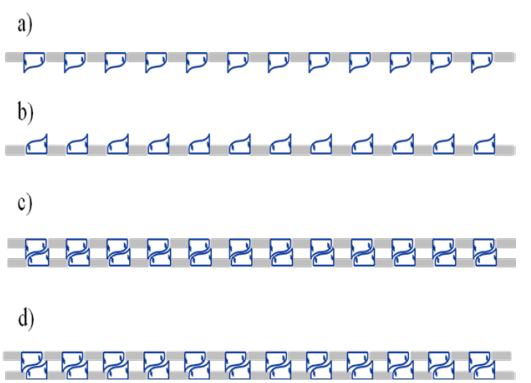

Fig. 2. Lateral view of the array: a) the phase mask, b) complex conjugate mask, c) pair of masks without lateral displacement, d) the pair with a lateral displacement.

Next, we use the asymmetric phase profile proposed by Alvarez, in reference 1, then

$$
\varphi(x, y)=-2 \pi(a / \lambda)\left(x^{3}+3 x y^{2}\right) /(\varepsilon d / 2)^{3} .
$$
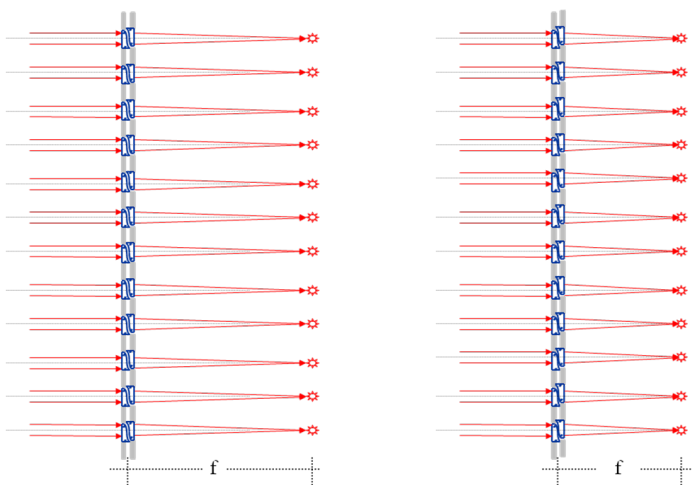

Fig. 3. A pair of masks for setting a lenslet array with variable focal length.

In Eq. (4) the letter "a" represents the maximum value of the optical path difference and $\lambda$ is the wavelength of the optical radiation. By substituting Eq. (4) in Eq. (3), we obtain that

$$
\begin{gathered}
t(x, y ; s)=K(s) \exp \left\{-i \pi\left[6(a / \lambda) s /(\varepsilon d / 2)^{3}\right]\left(x^{2}+y^{2}\right)\right\} \\
\operatorname{rect}[x /(\varepsilon-s / d) d] \quad \operatorname{rect}(y / \varepsilon d) .
\end{gathered}
$$

In Eq. (5), we employ the following definition $K(s)=\exp \left[-i \pi(a / 2 \lambda)(s / \varepsilon d / 2)^{3}\right]$.

We recognize that Eq. (5) represents the complex amplitude transmittance of a lens with a variable focal length, $f_{\text {var }}=(\varepsilon d / 2)^{3} / 6 a s$, as depicted in Fig. 3 .

Next, as depicted in Fig. 4a) and 4b), we consider the use of two standard lenslet arrays for implementing an array of telecentric optical processors. Now, we locate the previously described phase conjugated pair (in Fig. 3), at the Fourier plane of the telecentric array processor. In other words, we implement an array of spatial filters.

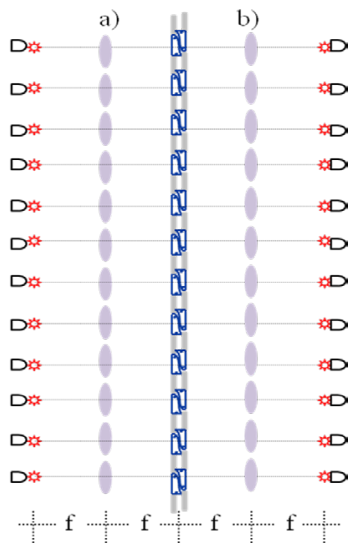

Fig. 4. Telecentric processor array: in a) and b) conventional lenslet arrays are used for implementing optically the Fourier transform, while at the Fourier plane the spatial filter is the pair in Fig. 3.

For this application, it is convenient to rewrite Equation 5 in terms of spatial frequencies. To this end, we employ the following new set of variables

$$
\begin{aligned}
& \mu=x / \lambda f, \quad v=y / \lambda f, \quad \sigma=s / \lambda f, \\
& \Omega=\varepsilon d / 2 \lambda f, \quad Q(\sigma)=K(s), \\
& W_{2,0}=-(3 a / \Omega) \sigma .
\end{aligned}
$$

In Eq. (6), we use the character $W_{2,0}$ for representing the wavefront aberration coefficient associated to a focal shift, or focus error. By substituting the variables of Eq. (6) in Eq. (5), we obtain that the generalized pupil function (of each element in the array processor) is

$$
\begin{aligned}
P(\mu, \nu ; \sigma)= & Q(\sigma) \exp \left[i 2 \pi\left(W_{2,0} / \lambda\right)\left(\mu^{2}+v^{2}\right) / \Omega^{2}\right] \\
& \operatorname{rect}(\mu / \Omega) \operatorname{rect}(v / \Omega) .
\end{aligned}
$$

Equation 7 takes into account the fact that at the Fourier domain (of any element of the optical array processor), we can keep the same pupil aperture $(\epsilon$ d) by using phase elements that are slightly larger than the pupil stops; for small values of $\sigma$. It is apparent from Eq. (7) that by using the processor array in Fig. 4, one can tune the amount of focus error by generating on the pair a lateral displacement $\sigma$. This feature has the following practical implication. Let us consider a set of point sources located at a distance $z_{0}=b f$ (with $0 \leq b \leq 1$ ) from the input focal plane of the first lenslet array (Fig. 4a). According to Newton's formula its image is at the distance $z_{1}=-f / b$, from the back focal plane of the same first lenslet array. Each image of the point source generates a paraxial spherical wave that impinges on the pupil aperture at the Fourier domain. The complex amplitude distribution of the paraxial wave is 


$$
u(x, y ; s)=\exp \left[i \pi(b / \lambda f)\left(x^{2}+y^{2}\right)\right]
$$

Thus, by comparison of Eq. (5) and Eq. (8), we claim that if $z_{1}=(\varepsilon d / 2)^{3} /(6 a s)$, then the wavefront leaving the pupil aperture is a plane wavefront, which focuses at the detector array located at the back focal plane of the second lenslet array (Fig. 4a). In other words, the processor array is useful for bringing into focus the points with

$$
b=(6 a f) s /(\varepsilon d / 2)^{3} .
$$

Therefore, the processor array is a tunable multiple axialscanning device.

Finally, we refer to Fig, 5 for describing the multiple use of the following phase profile

$$
\begin{aligned}
& \varphi(x, y)=2 \pi(a / \lambda) \\
& \left(x^{4}+4 x y^{3}-x(\varepsilon d / 2)^{2}\right) /(\varepsilon d / 2)^{3} .
\end{aligned}
$$

Eq. (10) is a 2-D version of the phase masks discussed in Ref. [7]. By substituting Eq. (10) in Eq. (3), we obtain that

$$
\begin{gathered}
t(x, y ; s)=\exp \left\{i 2 \pi\left[4(a / \lambda) s /(\varepsilon d / 2)^{4}\right]\left(x^{3}+y^{3}\right)\right\} \\
\exp \left\{i 2 \pi\left[(a / \lambda) /(\varepsilon d / 2)^{4}\right]\left(s^{2}-\varepsilon^{2} d^{2} / 2\right) x s\right\} \\
\operatorname{rect}[x /(\varepsilon-s / d) d] \operatorname{rect}(y / \varepsilon d) .
\end{gathered}
$$

It is apparent from the first phase term in Eq. (11) that one can generate a tunable cubic phase profile (both in $\mathrm{x}$ and $y)$. However, the second term introduces a spurious phase delay that is linear in $\mathrm{x}$, which can be reduced if $0 \leq s \leq \varepsilon d / \sqrt{2}$.

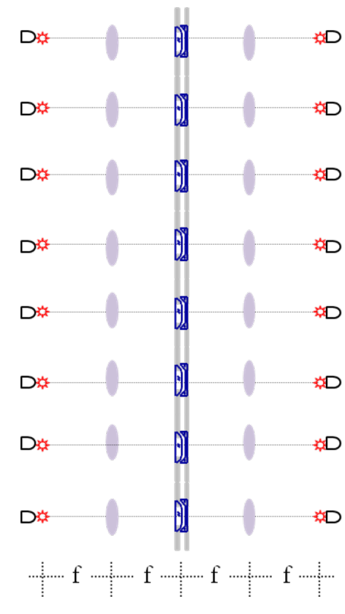

Fig. 5. As in Fig, 4, but for the phase screens in Ref. [6].

Summarizing, we have suggested the use of two classical lenslet arrays for setting a telecentric processor array. We have proposed to employ as a spatial filter, a pair of nonconventional arrays for controlling the focal length; and in this manner one has a multiple axialscanning device. Furthermore, we have identified another phase conjugate pair that is useful for tuning the depth of the field of a telecentric processor array.

\section{References}

[1] L.W. Alvarez, U.S. Patent 3,305,294 (3rd Dec. 1964)

[2] A.W. Lohmann, Italian Patent 727,848 (19th June, 1964).

[3] A.W. Lohmann, Appl. Opt. 9, 1669 (1970).

[4] I. A. Palusinski, J. M. Sasián, and J. E. Greivenkamp, Appl. Opt. 38, 86 (1999).

[5] N. López-Gil, H.C. Howland, B. Howland, N. Charman, R. Applegate, J. Opt. Soc. Am. A 15, 2563 (1998).

[6] E. R. Dowski, Jr., T.W. Cathey, Appl. Opt. 34, 1859 (1995).

[7] J. Ojeda-Castaneda, J.E.A. Landgrave, C.M. Gómez-Sarabia, Appl. Opt. 47, (2008)

[8] J.M. Burch, D.C. Williams, Appl. Opt. 16, 2445 (1977).

[9] A.W. Lohmann, D.P. Paris, Appl. Opt. 6, 1567 (1967).

[10] E. Acosta, S. Bará, J. Opt. Soc. Am. A, 22, 1993 (2005)

[11] S. Bernet, M. Ritsch-Marte, Appl. Opt. 47, 3722 (2008).

[12] S. Bará, Z. Jaroszewicz, A. Kołodziejczyk, V. Moreno, Appl. Opt. 30 1258 (1991). 